\title{
Influence of the cage on the migration and distribution of lubricating oil inside a ball bearing
}

\author{
He LIANG*, Yu ZHANG, Wenzhong WANG \\ School of Mechanical Engineering, Beijing Institute of Technology, Beijing 100081, China \\ Received: 27 November 2020 / Revised: 11 January 2021 / Accepted: 08 March 2021 \\ (C) The author(s) 2021.
}

\begin{abstract}
The migration and distribution of lubricant oil in a rolling bearing strongly affect the elastohydrodynamic lubrication performance between the balls and rings. However, oil re-lubrication is highly dependent on the bearing design, which is different from the ball-on-disc model. This study directly observed the distribution of the lubricant film in a custom-made model-bearing rig, with an outer ring replaced by a glass ring to allow full optical access. The influence of the cage type and surface properties were presented. The physical origin of the re-lubrication mechanism, including capillary flow and mechanical redistribution, was discussed.
\end{abstract}

Keywords: ball bearings; oil distribution; lubrication; cage

\section{Introduction}

Lubricating performance is intrinsically linked to the stable and long-life operation of rolling bearings. The inlet oil supply layer has been found to be one of the most important factors that ensures a thick film at the contact to separate the tribo-pair as it determines the degree of starvation. Pioneer researchers have mainly focused on the prediction of film thickness and the replenishment mechanism under starvation conditions. Wedeven et al. [1] experimentally determined the relationship between the position of the inlet meniscus and the film thickness reduction. Chevalier et al. [2] assumed a layer of oil with a specific film thickness in his numerical model to determine the film thickness under starvation conditions, and then discussed the influence of different inlet film shapes. Liang et al. $[3,4]$ proved experimentally that the asymmetrical inlet oil reservoir caused by centrifugal effects could lead to partial starvation in the contact. Oil reflow was the key to replenishing the inlet oil reservoir and maintaining long-life lubrication. Chiu [5] first explained that replenishment was driven by surface tension and hindered by viscosity and speed. Jacod et al. [6] found that replenishment mainly occurred near the contact driven by capillary forces. Other factors, such as centrifugal effects [4], angled surface velocities [7], wettability [8], and oil-air lubrication [9], have also been discussed to enhance the understanding of oil replenishment. All the above mentioned studies were mainly based on a single ball-on-disc point model.

In contrast to the established film thickness rules applied to the ball-on-disc model, the lubrication in a rolling bearing is less understood. In practice, the lubrication inside a rolling bearing is quite different from that of a single ball-on-disc model; hence, it is difficult to directly apply single-point lubrication theories to rolling bearings. Some studies have shown that the oil distribution inside the bearing is not uniform [10, 11], and oil replenishment driven by surface tension rarely occurs in a rolling bearing [12]. Film formation in the contact is directly related to the shape and thickness of the inlet supply oil layer, which is apparently dependent on the bearing design, including the bearing size, cage design, and number of balls. The cage is an important component of bearings used to isolate and guide the rolling elements. Some

* Corresponding author: He LIANG, E-mail: lianghe@bit.edu.cn 
studies have shown that different cage clearance values [13] and cage geometries [14, 15] can significantly influence the migration and distribution of lubricating oil inside the rolling bearing. Damiens et al. [13] studied the effect of cage clearance on the lubricant supply and elastohydrodynamic (EHL) film thickness in a ball-on-disc device. They mounted a single cage pocket on the ball, and showed that decreasing the cage clearance could scrape and reduce the inlet film in the case of oil lubrication as well as redistribute the grease to increase the EHL film thickness. The experimental results by Cann and Lubrecht [14] showed that the cage design could have both positive and negative effects on lubricant redistribution within a bearing. In addition, grease could be over-rolled and sheared to release oil in the cage pockets [16], whose structure was changed and influenced film formation at different speeds [17].

One of the most important factors that determine the lubrication level in rolling bearings is the supply of lubricant to the contact zone. If the oil distribution in the bearings can be investigated directly, then information can be obtained on inlet oil supply conditions, thus providing lubrication conditions in the contact. For this purpose, the migration and distribution of oil in a model rolling element bearing were observed using optical methods in this study. The outer ring was replaced by a glass ring to provide an optical window, while the oil was tagged with a fluorescent dye. The influence of the cage design on the supply of lubricant to the contact zone was discussed to study the migration and distribution of the lubricant film in ball bearings. The lubrication distribution at low speeds was first investigated. Therefore, the centrifugal effects were not considered. As the majority of rolling bearings are lubricated with grease, which is widely accepted to bleed oil for lubrication, the study of oil lubrication presented in this paper can also shed light on certain aspects of the oil supply and replenishment mechanisms in grease-lubricated bearings, thereby contributing to the further development of the grease lubrication theory.

\section{Apparatus}

A custom ball bearing rig was built and used in this study, as shown in Fig. 1. The outer ring was replaced by a flat glass ring to allow the observation of the oil distribution inside the ball bearing. The test bearing was a commercial angular contact ball bearing 7014 . As the study focused on the influence of cages on bearing lubrication, three different types of cages were used: cylindrical cage, conical frustrum cage, and asymmetrical cylindrical cage. The specific cage parameters are listed in Table 1. Both the cylindrical cage and conical frustum cage for tests 1 and 2 were obtained from commercial bearings. The asymmetrical cylindrical cage for test 3 was created by cutting a portion of the cylindrical cage. An oleophobic coating was applied to the outer surface of the cylindrical cage for test 4 . Figure 2 compares the dimensions

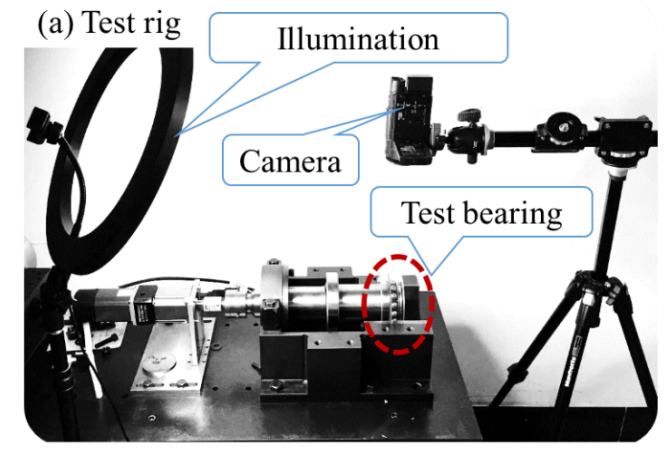

(b) Left view

(c) Front view

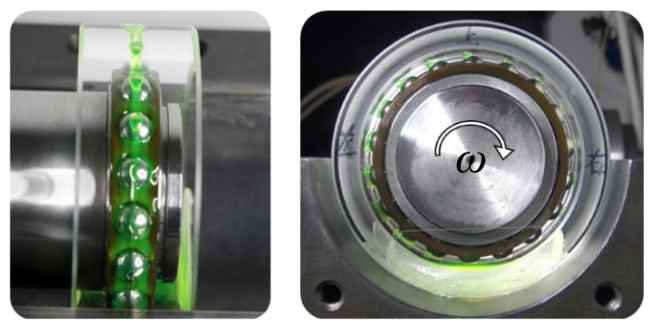

Fig. 1 A diagram of the test ball bearing and images taken from the left view and front view.

(a) $4.17 \mathrm{~mm}$

(b) $0.37 \mathrm{~mm}$
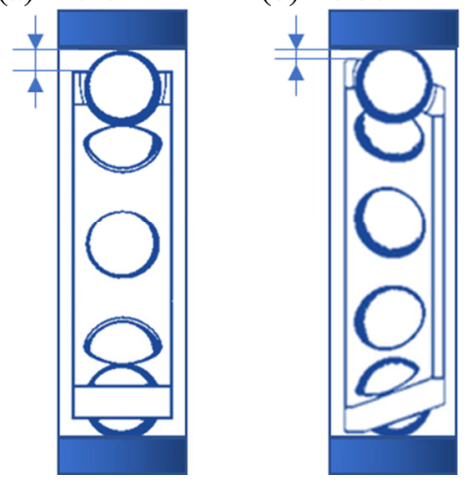

Fig. 2 Dimensions of different cages used for a ball bearing: (a) cylindrical cage and (b) conical frustum cage. 
Table 1 Shape and parameters of the tested cages.

\begin{tabular}{cccc}
\hline Cage & Material & Number of pockets & Surface modification \\
\hline Cylindrical cage & Polyamide 66 & 18 & None \\
Conical frustum cage & Polyamide 66 & 18 & Nolyamide 66 \\
Asymmetrical cylindrical cage & Polyamide 66 & 18 & None \\
Cylindrical cage & & With oleophobic & coating \\
\hline
\end{tabular}

of the different cages for a ball bearing. For the cylindrical cage, the clearance between the cage and the outer ring was $4.17 \mathrm{~mm}$. For the conical frustum cage, the clearance between the cage and the outer ring changed along the bearing width. The narrowest gap was $0.37 \mathrm{~mm}$ on the left side.

The ball bearing was loaded radially downward, exerting a maximum Hertzian contact pressure of 1.47 GPa. The outer ring remained static, and the inner ring rotated to drive the balls and cage at a room temperature of $23 \pm 2{ }^{\circ} \mathrm{C}$. Quantitative lubricating oil was injected into the bottom of the bearing to create an oil supply bath. A synthetic oil with a viscosity and surface tension of $539 \mathrm{mPa} \cdot \mathrm{s}$ and $26.38 \mathrm{mN} / \mathrm{m}$, respectively, was used in all the tests. To observe the oil distribution, the oil was tagged with a fluorescent dye.

\section{Results}

\subsection{Comparison of oil migration using two types of commercial cages}

Figure 3 shows the left and front views of the migration of oil along with the running time for the cylindrical cage, as seen in Fig. 1. The images in Fig. 3 were taken under the following conditions: inner ring revolution of $5 \mathrm{rpm}$ and oil supply of $4 \mathrm{~mL}$ and were taken as the rotational speed of the inner ring reached the target velocity of $5 \mathrm{rpm}$, and the moment was set to $0 \mathrm{~s}$. At the initial state, all the oil was at the bottom oil supply bath, and no oil was distributed to the other elements or places in the ball bearing. After the bearing started to rotate, an elliptical oil reservoir was formed between the balls and the static outer ring at $0 \mathrm{~s}$, as shown in Fig. 3. The oil reservoir moved upward along the outer raceway, following the revolving balls. The size of the oil reservoir at a fixed position also increased with time, and then reversed and began to decrease at $80 \mathrm{~s}$. This may be attributed to the insufficient oil supply at the bottom oil bath as oil slowly migrated to other elements in the ball bearing. An oil band also formed on the outer raceway with a width similar to that of the neighboring oil reservoir. As the outer ring was static, the oil in the oil band should be transferred from the oil reservoir. At $160 \mathrm{~s}$, the oil reservoir shrank obviously, and the oil dispersed on the cage could be distinguished as it was mainly deposited between adjacent pockets.

To observe the oil distributed on the cage, 12 balls were removed, and only six were left and evenly placed along the circumferential direction, as shown in Fig. 4. It can be observed that oil accumulated at the rear edge of the pocket hole, indicating that the pocket actually acted as a scraper. The scraped oil flowed downward along the cage, slowly driven by gravity. As the running time increased, the volume of scraped oil also increased. Compared with the case of 18 balls, the volume of oil on the cage was greater while the oil reservoir was hardly seen near the ball-outer ring contact when only six balls were left. This indicates that the oil in the oil reservoir and on the cage are actually in competition. The rear edge of the pockets could inevitably scrape the oil from the ball owing to gravity. The reduced oil carrying capacity is due to the lack of balls that carry and pump the oil upward, leading to the reduction of the oil reservoir size. 
Figure 5 shows the left and front views of the migration of oil against the running time for the conical frustum cage at $5 \mathrm{rpm}$ and $4 \mathrm{~mL}$ of oil supply. It can be seen in Fig. 5(a) that the variations in oil reservoir size gradually increased and then decreased with running time, similar to the trend in the cylindrical cage test. A significant difference is that an oil string appeared at the left end of the cage and climbed together with the oil reservoir. When the oil string connected with the oil reservoir, it created an asymmetric elliptical oil reservoir, as seen at 20 and $40 \mathrm{~s}$ in Fig. 5(a). The oil string not only changed the


Fig. 3 Migration of oil against running time for the asymmetrical cylindrical cage (4 mL, $5 \mathrm{rpm})$.
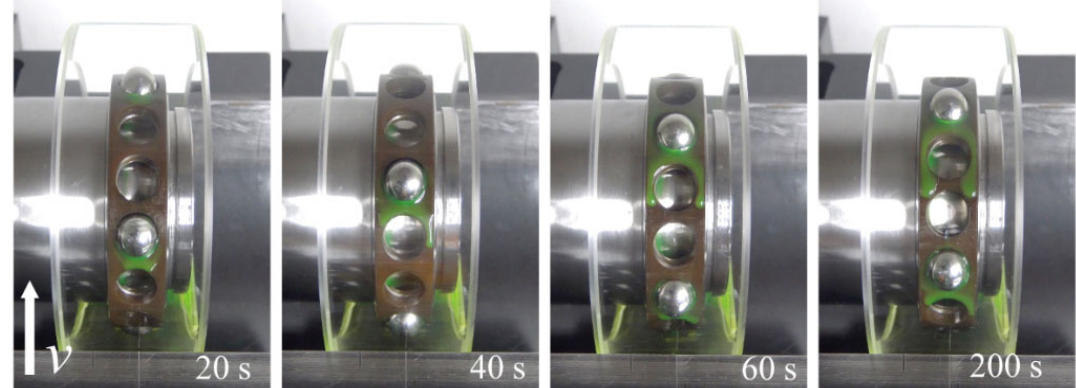

Fig. 4 Oil distributed on the cylindrical cage in the ball bearing with six balls (4 mL, $5 \mathrm{rpm})$.
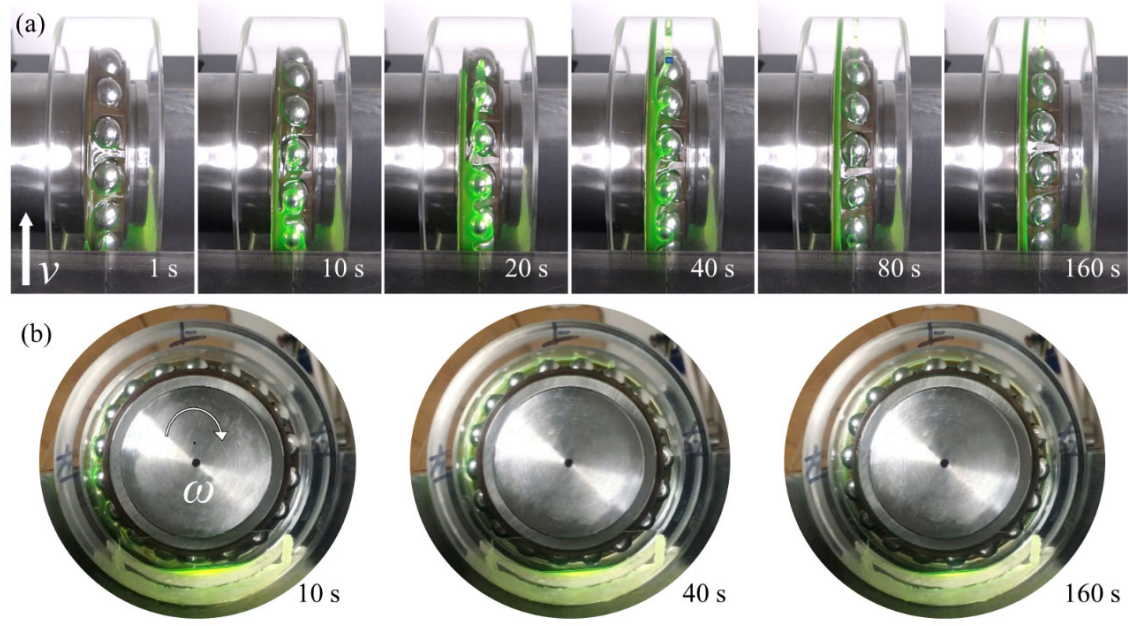

Fig. 5 Migration of oil against running time using a cylindrical cage (4 mL, $5 \mathrm{rpm}$ ). 
shape of the oil reservoir but also its size. At $40 \mathrm{~s}$, the size of the oil reservoir began to decrease significantly, while the oil string remained long and thick. At 80 and $160 \mathrm{~s}$, the oil reservoir shrank and was hardly seen, while the oil string still existed. Comparing the dimensions of the ball bearing in Fig. 2, we found that the existence of the oil string on the left side was due to the narrow gap between the outer ring and the conical frustum cage. The strong capillary force in the narrow gap caused the oil string to flow with the revolution of the cage. The oil string was continuous, while the oil reservoir formed by the outer ring and the rolling balls was spaced for each ball, which made it harder for oil to flow from one reservoir to another. As most of the oil accumulated at the oil string, there was much less oil in the reservoir or on the cage surface for the ball bearings in the conical frustum cage. Although the oil was sufficiently distributed in the ball bearing in the form of an oil string, it was difficult to lubricate the ball-ring contact. Thus, the oil is not effective for lubrication.

\subsection{Comparison of oil reservoir's dimension for different cages}

The above experimental results suggest the existence of competition for oil adhered to different elements or different places; for example, oil in the oil reservoir or oil on the cage. In the following test, a portion of the cage was cut to obtain an asymmetrical cylindrical cage, as shown in Table 1 and Fig. 6. The idea is to reduce the oil adhered to the cage by reducing the surface area of the cage, and to check whether more oil can accumulate in the oil reservoir by analyzing the change in the oil reservoir size. A comparison of the oil reservoirs for the two cages in Figs. 3 and 6 show that the size of the oil reservoir for the asymmetrical cylindrical cage is similar to that of the cylindrical cage from 0 to $40 \mathrm{~s}$; however, it was slightly larger after $80 \mathrm{~s}$. The comparison results suggest that cutting the cage to reduce the surface area could help increase the oil in the reservoir as well as the oil adhered to the cage. As a result, more oil was left or flowed back to the bottom oil bath and continuously supplied the oil reservoir.

To further study the influence of cage type on the size of the oil reservoir near the contact zone, a dimensionless oil reservoir width $(W)$ is defined as follows:

$$
W=\frac{w}{D}
$$

where $w$ is the maximum width of the oil reservoir, and $D$ is the diameter of the rolling ball. Figure 7 shows the change in $W$ at top point of the ball bearing with respect to running time. For all cage types, the $W$ at the top point of the ball bearing rapidly increased and then slowly decreased until reached a stable state. The $W$ for the conical frustum cage was much smaller than that for the cylindrical and asymmetrical cylindrical cages. This was attributed to the oil string that formed between the outer ring and the cage at the large end. The $W$ for the conical frustum cage was the quickest to attain stability, and was maintained at a small value of approximately 0.1 . The $W$ for the

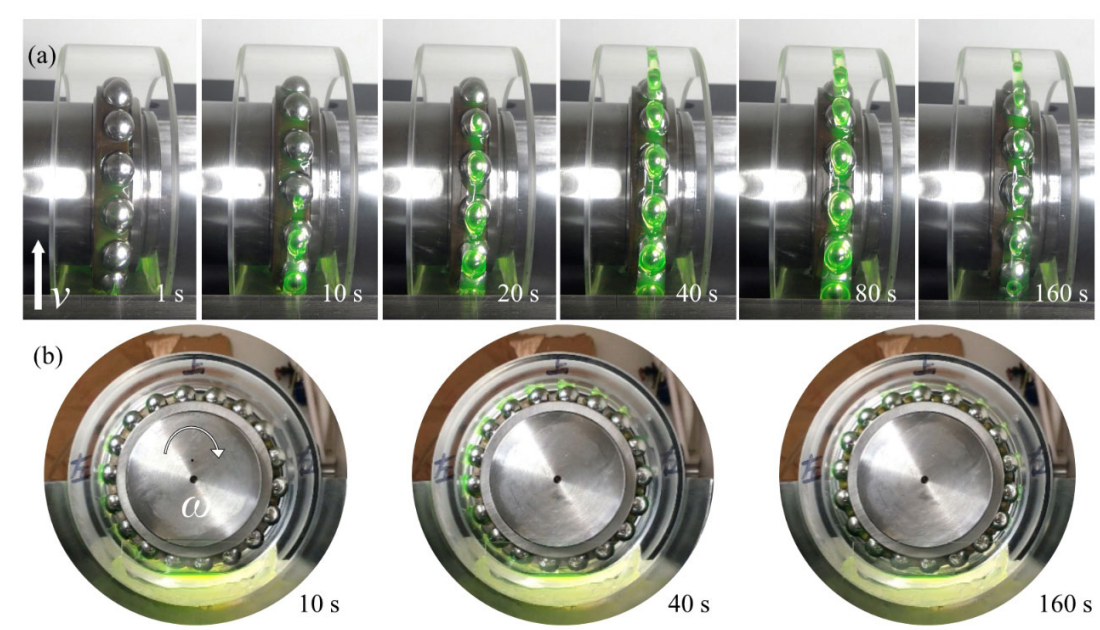

Fig. 6 Migration of oil against running time for the conical frustum cage (4 mL, $5 \mathrm{rpm}$ ). 


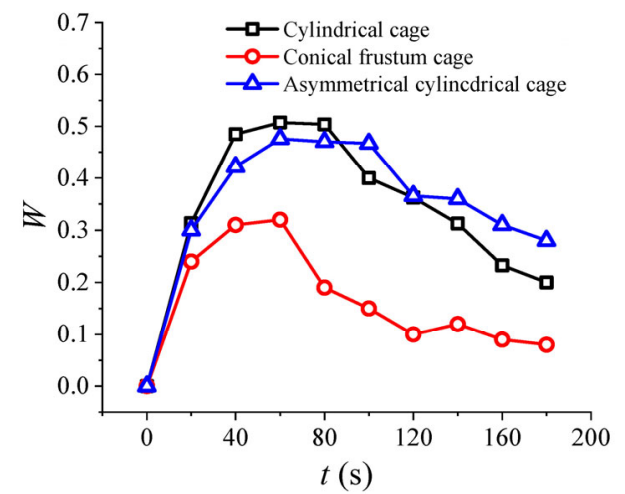

Fig. 7 Comparison of dimensionless oil reservoir width against the running time for the three types of cages ( $4 \mathrm{~mL}, 5 \mathrm{rpm})$.

asymmetrical cylindrical cage was quite close to that for the cylindrical cage. It increased gradually from 0 to $80 \mathrm{~s}$, and then decreased slowly, resulting in a larger oil reservoir compared with that of the cylindrical cage after $80 \mathrm{~s}$. The results confirmed the competition

(a) Initial

CA left: $31.9^{\circ}$, CA right: $31.9^{\circ}$ for oil in ball bearings. Cutting a portion of the cage could help maintain the oil concentration at the oil reservoir to re-lubricate the ball-ring contact to some extent. However, the oil carrying capacity decreased, leading to a slower rise in $W$ for the asymmetrical cylindrical cage.

Another comparison test was designed to apply an oleophobic coating to the surface of the cylindrical cages. The contact angles were tested for both cages, as shown in Fig. 8. The contact angle on the cylindrical cage was $31.9^{\circ}$, which increased to $51.2^{\circ}$ with the application of oleophobic coating.

Figure 9 shows the left view of the oil distribution in the ball bearing using a cylindrical cage with and without oleophobic coating. The conditions were $5 \mathrm{rpm}$ and an oil supply of $7 \mathrm{~mL}$. From the initial time to $60 \mathrm{~s}$, the size of the oil reservoirs did not show a significant difference between the two cages.

(b) With oleophobic coating

CA left: $51.2^{\circ}$, CA right: $51.2^{\circ}$
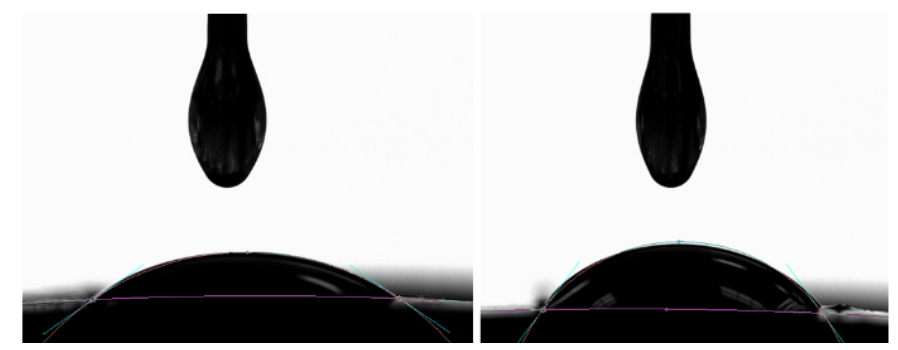

Fig. 8 Measured contact angle on the cylindrical cage without and with oleophobic coating.


Fig. 9 Comparison of oil distribution in the ball bearing using a cylindrical cage: (a) without and (b) with oleophobic coating (7 mL, $5 \mathrm{rpm}$ ). 
However, it gradually increased for the oleophobiccoated cage from 60 to 240 s. It can be seen that before the oleophobic coating was applied, there was abundant oil adhering to the surface of the cage, which was clearly seen after $120 \mathrm{~s}$. After the oleophobic coating was applied, oil was still deposited on the surface of the cage as the oil supply was sufficient; however, more oil was accumulated between pockets with a narrower width than the diameter of the pocket hole. The test results show that reducing the oil in the cage effectively increased the oil volume near the ballouter ring contact, which is beneficial for improving the lubrication performance between the ball-outer ring contact.

Figure 10 compares the $W$ at the top point in the ball bearing for the cylindrical cage with and without the oleophobic coating. The rising region for the two cages acted similarly as the curve overlapped with each other from the initial time to $50 \mathrm{~s}$. However, the $W$ for the uncoated cage immediately decreased after it reached the maximum value, and achieved a stable state with a very small oil reservoir. The $W$ with oleophobic coating could remain at the top platform for another $40 \mathrm{~s}$ and then started to decrease. This resulted in a much larger oil reservoir for the cylindrical cage with an oleophobic coating.

\subsection{Distribution of oil reservoir influenced by different cages}

Figure 11 shows the typical shapes of an oil reservoir near the ball-outer ring contact formed for different

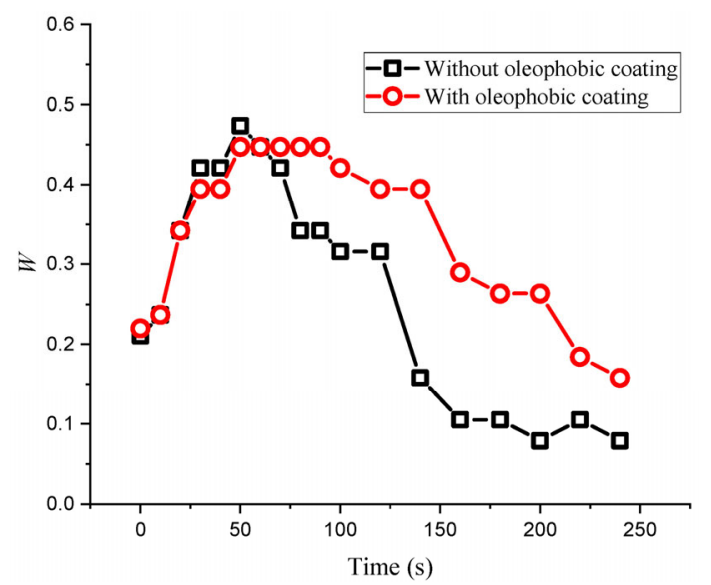

Fig. 10 Comparison of dimensionless width of oil reservoir against running time using cylindrical cages without and with oleophobic coating ( $7 \mathrm{~mL}, 5 \mathrm{rpm})$.

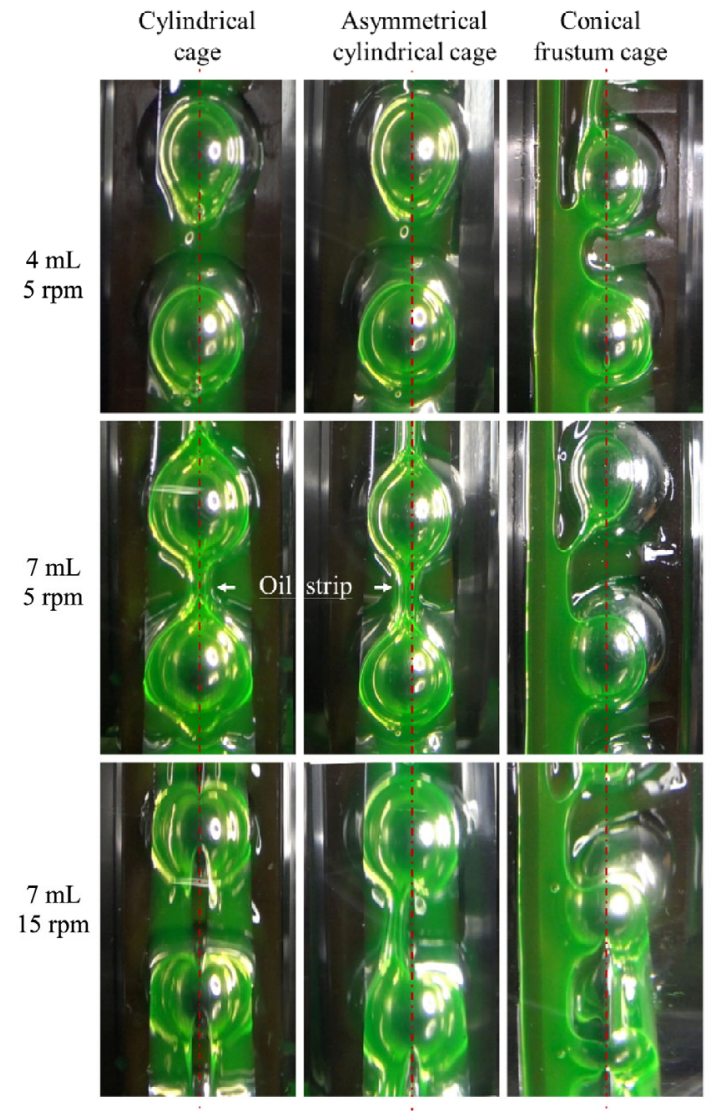

Fig. 11 Typical distribution of oil in the ball bearing using different types of cages.

types of cages under three conditions (from the left view). At $4 \mathrm{~mL}$ and $5 \mathrm{rpm}$, the oil reservoirs for both the cylindrical and asymmetrical cylindrical cages were elliptical. Owing to the sufficient accumulation of lubricating oil on the large side of the conical frustum cage, the oil reservoir was connected to the oil string; therefore, the adjacent oil reservoirs were also connected through the oil string. When the oil supply increased from 4 to $7 \mathrm{~mL}$, and the rotational speed of the inner ring was maintained at $5 \mathrm{rpm}$, an oil strip appeared connecting the adjacent oil reservoir for the cylindrical and asymmetrical cylindrical cages. The oil strip could move by following the revolution of the oil reservoir and replenish the oil reservoir. When the rotational speed increased from 5 to $15 \mathrm{rpm}$ at $7 \mathrm{~mL}$, the oil reservoir diverged at the outlet zone and formed a typical butterfly shape for the cylindrical cage. The oil reservoir for the cylindrical cage remained symmetrical at all oil supply and rotational speeds. For the asymmetrical cylindrical cage whose right side was cut, the oil reservoir was asymmetric, as there 
was an oil strip connecting the adjacent oil reservoir only on the left side and not on the right side. This indicates that there was more oil on the left than on the right side. For the conical frustum cage, the oil reservoir showed a similar shape, but always had a tail connecting the oil string on the left side. The oil reservoir using the conical frustum cage showed a large volume; however, the inlet distance (i.e., the distance from the inlet meniscus to the center of the Hertzian contact along the middle cross-section) was less than that of the elliptical oil reservoir using the other two cages. These results show that the cage had the ability to redistribute the oil deposited in the ball bearing.

\section{Discussion}

\subsection{Competition of lubrication oil in a ball bearing}

It is easy to determine whether there is competition for the oil on different elements in a ball bearing. If the oil on the cage is reduced, then the oil in the oil reservoir can be replenished. The experimental results show that there are two main forms of oil on the cage, which can hardly replenish the oil reservoir. On one hand, the oil is widely spread on the surface of the polymer cage, especially near the rear edge of the pocket. On the other hand, oil could accumulate in the small gap that formed between the outer ring and the cage, as shown in Fig. 5. It can be reasonably expected that oil could also accumulate in the small gap between the inner groove and the cage, although this was not directly observed in our tests. Figure 12 shows the left view of the oil distribution in the conical frustum cage for three different amounts of oil supplied. The images in Fig. 12 were taken at $10 \mathrm{rpm}$ after running for $30 \mathrm{~s}$. The left oil string gradually grew against the oil supply; however, the size of the oil reservoir did not show a distinct increase. This implies that most of the oil accumulated in the continuous oil string and increasing the oil supply did not increase the oil reservoir.

Figure 13 compares the dimensions of the small gap that formed between the conical frustum cage and the outer ring at the large end, and the maximum oil film thickness in the oil reservoir. The dimensions of the gap were measured directly as $0.37 \mathrm{~mm}$. The



Fig. 12 Oil distribution for the conical frustum cage at $10 \mathrm{rpm}$ for oil supplies ranging from 1 to $7 \mathrm{~mL}$.

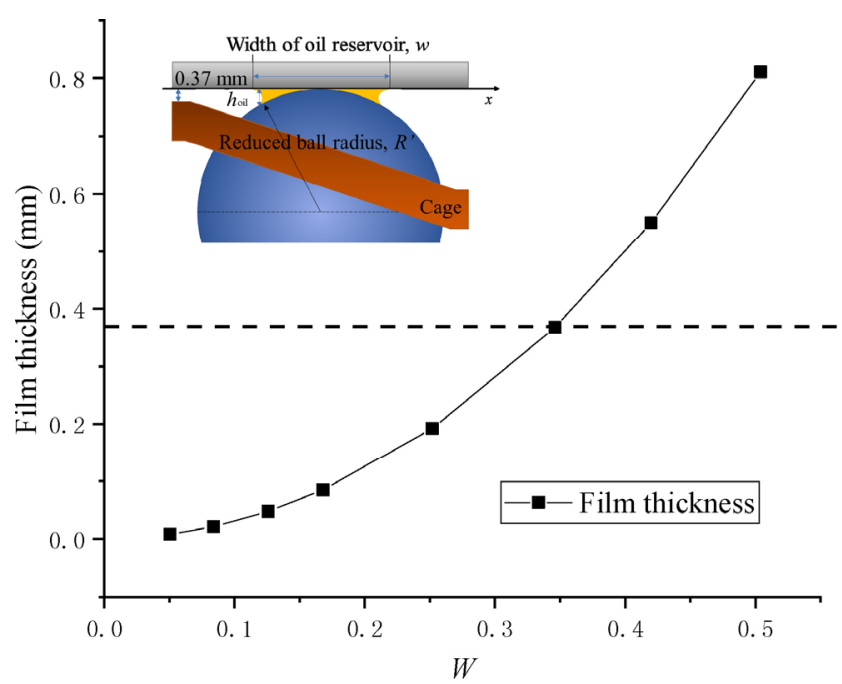

Fig. 13 Maximum film thickness against the dimensionless width of the oil reservoir for the conical frustum cage.

maximum oil film thickness in the oil reservoir was calculated to be the same as the gap between the outer ring and the rolling ball at the inlet meniscus. Figure 13 shows that the maximum film thickness in the oil reservoir increased with the dimensionless width. When $W$ was approximately 0.35 , the maximum film thickness was $0.37 \mathrm{~mm}$, which is the value of the gap between the outer ring and the conical frustum cage at the left end. Compared with Fig. 7, it is interesting to find that 0.35 was also the maximum value of $W$ that could be reached. Figure 7 shows the $W$ at the top point in the ball bearing, where the oil reservoir diverged from the oil string on the left side, as shown in Fig. 5. The oil reservoir could achieve a dimensionless width larger than 0.35 at a lower point 
if it was linked to the left oil string. The oil was driven by capillary force, which was influenced by the gap clearance. When the oil reservoir diverged from the oil string, it resulted in a similar thickness for both the oil string and the maximum film thickness in the oil reservoir owing to the balanced capillary force.

\subsection{Role of the cage pocket for lubrication}

The results show that the cage has multiple effects on lubrication. On one hand, the small clearance between the ball and the pocket is a good place to store lubricants, thus replenishing the oil on the ball. The comparison tests of the asymmetrical cylindrical cage showed that the oil carrying capacity slightly decreased if a portion of the pocket was cut because the $W$ increased at a slightly slower rate than that of the cylindrical cage against running time. The maximum value of $W$ was also slightly lower than that of the cylindrical cage. In contrast, the rear edge of the pocket acted as a scraper to redistribute the oil. Under the force of gravity, the balls inevitably touched the lower edge of the pocket. As a result, the oil on the ball was first transferred to the cage, and then replenished the next ball. This significantly reduced the oil on the ball and may be beneficial for averaging the oil for different balls.

\subsection{Improvement for the oil supply to the ball- outer ring contact}

As lubrication of the contact between the ball and the ring is quite important, it is worth modifying the cage to obtain a more sufficient oil supply for the ball-outer ring contact. A reasonable design of the cage structure could help accumulate the oil near the ball-ring contact. The gap between the large end of the conical frustum cage and the outer ring is so small that it attracts a large amount of oil, which weakens the oil flow to the ball-ring contact region. This paper sheds light on the modification of cages for improved lubrication. One way is to optimize the cage structure to prevent the small gap between the cage and rings, or to narrow the cage width to reduce its surface area. Another is to apply an oleophobic coating on the surface of the cage, which also provides a more effective lubrication for the ball-outer ring contact.

\section{Conclusions}

This study investigated the influence of different cage shapes and surface properties on the migration and distribution of lubricants based on a model ball bearing. The oil reservoir formed between the rolling balls and the outer ring, and the oil that adhered to the cage and the outer ring was observed directly by replacing the outer ring with a glass ring. The conclusions are as follows:

1) The lubricating oil on different bearing elements in a ball bearing is the result of oil competition.

2) The efficient re-lubrication for the ball-outer ring contact is significantly influenced by the cage shape and cage surface properties. The ineffective oil on the cage can be reduced by changing the cage geometry from a conical frustum cage to a cylindrical cage, reducing the width of the cage, and applying an oleophobic coating, to increase the oil supply for the ball-outer ring contact.

3) The cage pocket has a dual effect on oil re-lubrication for the ball-outer ring contact. The microgap between the ball and cage pocket is important for oil storage owing to the capillary force. The cage pocket also acts as a scraper to reduce oil on the ball. The reasonable design of the clearance between the cage pockets and balls can enhance the capillary force and control the migration ability of the lubricant.

\section{Acknowledgements}

The project was supported by the National Key R\&D Program of China (2018YFB2000604), the National Natural Science Foundation of China (51805031 and U1637205), and Tribology Science Fund of State Key Laboratory of Tribology (SKLTKF18A01).

Open Access This article is licensed under a Creative Commons Attribution 4.0 International License, which permits use, sharing, adaptation, distribution and reproduction in any medium or format, as long as you give appropriate credit to the original author(s) and the source, provide a link to the Creative Commons licence, and indicate if changes were made.

The images or other third party material in this article are included in the article's Creative Commons licence, unless indicated otherwise in a credit line to 
the material. If material is not included in the article's Creative Commons licence and your intended use is not permitted by statutory regulation or exceeds the permitted use, you will need to obtain permission directly from the copyright holder.

To view a copy of this licence, visit http://creativecommons.org/licenses/by/4.0/.

\section{References}

[1] Wedeven L D, Evans D, Cameron A. Optical analysis of ball bearing starvation. J Tribol 93(3): 349-361 (1971)

[2] Chevalier F, Lubrecht A A, Cann P M E, Colin F, Dalmaz G. Film thickness in starved EHL point contacts. J Tribol 120(1): 126-133 (1998)

[3] Liang H, Guo D, Luo J B. Experimental investigation of lubrication film starvation of polyalphaolefin oil at high speeds. Tribol Lett 56(3): 491-500 (2014)

[4] Liang H, Guo D, Ma L R, Luo J B. Experimental investigation of centrifugal effects on lubricant replenishment in the starved regime at high speeds. Tribol Lett 59(1): 1-9 (2015)

[5] Chiu Y P. An analysis and prediction of lubricant film starvation in rolling contact systems. S L E Trans 17(1): 22-35 (1974)

[6] Jacod B, Pubilier F, E Cann P M, Lubrecht A A. An analysis of track replenishment mechanisms in the starved regime. Tribol Ser 36: 483-492 (1999)

[7] Li X M, Zhou G Y, Guo F, Wang X B, Zhang J J. Enhanced lubricant replenishment by angled surface velocities in EHL contacts. Tribol Int 145: 106132 (2020)

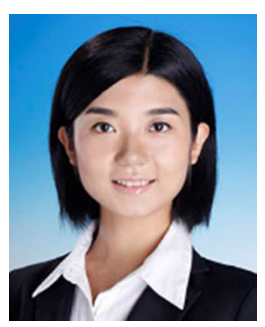

He LIANG. She received her B.S. degree in 2010 and Ph.D. degree in 2015, respectively, from Tsinghua University. She worked as a postdoc in Tribology group at Imperial College London from 2015 to 2018.

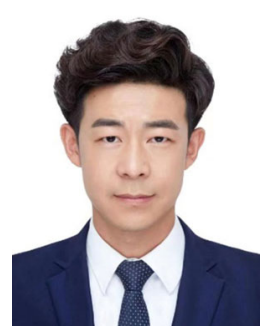

Yu ZHANG. He received his M.S. degree in mechanical engineering in 2019 from Beijing Institute of
[8] Liu C L, Guo F, Wong P L, Li X M. Tribological behaviour of surfaces with stepped wettability under limited lubricant supply. Tribol Int 141: 105880 (2020)

[9] Liang H, Guo D, Ma L R, Luo J B. The film forming behavior at high speeds under oil-air lubrication. Tribol Int 91: 6-13 (2015)

[10] Farrall M, Hibberd S, Simmons K. The effect of initial injection conditions on the oil droplet motion in a simplified bearing chamber. J Eng Gas Turbines Power 130(1): 012501 (2008)

[11] Wu M X, Wu W, Yuan S H, Hu J B. Study of multiplepoint oil-jet lubrication of high-speed ball bearings. Power Transmission Engineering 6: 44-46 (2014)

[12] Gershuni L, Larson M G, Lugt P M. Lubricant replenishment in rolling bearing contacts. Tribol Trans 51(5): 643-651 (2008)

[13] Damiens B, Lubrecht A A, Cann P M. Influence of cage clearance on bearing lubrication. Tribol Trans 47(1): 2-6 (2004)

[14] Cann P E, Lubrecht A A. Bearing performance limits with grease lubrication: The interaction of bearing design, operating conditions and grease properties. J Phys D: Appl Phys 40(18): 5446-5451 (2007)

[15] Cen H, Lugt P M. Replenishment of the EHL contacts in a grease lubricated ball bearing. Tribol Int 146: 106064 (2020)

[16] Lugt P M. A Review on Grease Lubrication in Rolling Bearings. Tribol T 52(4): 470-480 (2009).

[17] Li X M, Guo F, Poll G, Fei Y, Yang P. Grease film evolution in rolling elastohydrodynamic lubrication contacts. Friction 9(1): 179-190 (2021)

She is now an assistant professor in the School of Mechanical Engineering, Beijing Institute of Technology, with research interests covering fundamental of lubrication theory, tribological applications in rolling element bearings, and test method.

Technology. His research interest is the lubrication of rolling bearing, especially the experimental study of distribution of lubricating oil. 


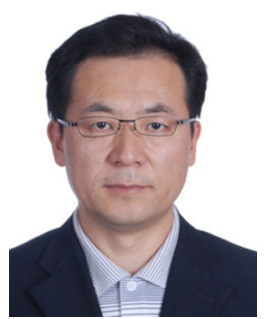

Wenzhong WANG. He received his Ph.D. degree in mechanical engineering from Tsinghua University, China, in 2003. He joined the School of Mechanical Engineering at Beijing Institute of Technology in
2006. His current position is a professor and director of the Department of Machine Design. His research areas cover modeling of lubricated contact problem, contact model for inhomogeneous and function gradient materials, dynamics of rolling bearing, and thermal analysis. 